\section{Management of Nasal Fractures}

\author{
Çiğdem Firat Koca ${ }^{1 *}$ and Ahmet Kizilay ${ }^{2}$ \\ ${ }^{1}$ Physician Malatya State Hospital, Malatya, Turkey \\ ${ }^{2}$ Department of Otolaryngology, Inonu University, Malatya, Turkey
}

\begin{abstract}
Nasal trauma has an important role in the field of craniofacial traumas. A thorough evaluation, including detailed examination and initial history, is necessary. The aim of this article is to focus on the management of nasal trauma, pathophysiology, diagnosis and
\end{abstract} treatment approaches.

Keywords: Nasal fracture; Septal fracture; Closed reduction; Open reduction

\section{Introduction}

Nasal fractures are the most common kind of facial fractures in adults and account for greater than $50 \%$ of all facial fractures $[1,2]$. The most frequent cause of fracture is blunt trauma to the midface. This kind of trauma frequently is a result of fighting, sports, and motor vehicle accidents $[2,3]$.

The nasal bone is consist of bones and cartilages, supporting the skeletal structure. This structural support is necessary for the aesthetic midface appearance and also for inspiration. To protect this framework an urgent evaluation is necessary. Initial examination may, sometimes, be difficult because of the diffuse nasal edema. Half of nasal fractures, often severe injuries, require reoperative rhinoplasty $[4,5]$.

Nasal fracture is less common in children under 5 years old, due to the less facial trauma exposure. There is an increasing frequency with age, especially between 16-20 years old boys [6]. Pure nasal fractures occured heavily among men under 25 years of age, and the major cause is fighting [7].

In children and women physical abuse should be remembered and be evaluated immidiately. Nasal fractures may be alone and/or with other facial fractures [8].

*Corresponding author: Çiğdem Firat KOCA, Malatya State Hospital, Malatya, Turkey, Tel: +90 5302239624; E-mail: cifirat@hotmail.com

Citation: Koca ÇF, Kizilay A (2015) Management of Nasal Fractures. J Emerg Med Trauma Surg Care 2: 007.

Received: February 15, 2015; Accepted: March 27, 2015; Published: April 09, 2015
Significant nasal injuries with septal and nasal shift cause nasal airway obstruction and require open treatment techniques, such as rhinoplasty [2].

\section{Anatomy}

The anatomic structure of nose is very important to understand the present pathophysiology. The maxilla, ethmoid, frontal, vomer and nasal bones form the bony structure of the nose. Most commonly, fractures occur distally where the nasal bones become thinner and broader. Proximal nasal bone injuries are considered nasoorbitoethmoid fractures, requires additional treatment (Figure 1) $[2]$.

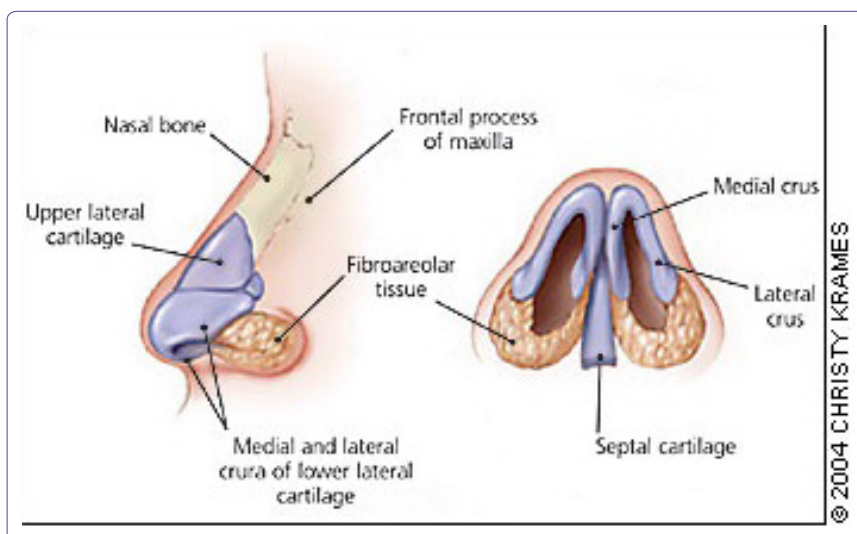

Figure 1: Anatomic relationship between the nasal bones, cartilage, and septum.

The paired upper and lower lateral cartilages and the septum form the cartilaginous nasal structure. In a midline position the quadrangular cartilage is formed by the upper lateral cartilages [9]. The transition zone between the thicker proximal and thinner distal segments in the lower one third to one half of the nasal bones is susceptible to fractures. Approximately $80 \%$ of nasal fractures occur at this transition zone. Trauma to the cartilaginous structures of the results in commonly dislocation, displacement, or avulsion injuries more than true fractures [10]. The upper lateral cartilages have fibrous articulations with the nasal bones superiorly, the quadrangular cartilage medially, and the lower lateral cartilages inferiorly. This articulation maintains resistance to inspiratory airflow, and this resistant area called internal nasal valve (Figure 2) [11].

The cartilaginous septum is posteriorly interposed between the perpendicular plate of the ethmoid and the vomer with a strong osseochondral junction. Weak areas of the cartilaginous dorsum and the weak connections between the septal cartilage and maxillary crest account for a high incidence of fracture- dislocation of the septum following nasal trauma [10]. The lower lateral portions responsible for the aesthetic contour of the nose. This strong cartilaginous structure is resistant to damage. The quadrangular cartilage forms the nasal septum. The thicker posterior septal cartilage supports the upper two-thirds of the nose. The septum is the major growth center of the face in chidren. This important task continues until ages 12 to 13 year. In all ages, this midline structure is supported laterally by the medial 


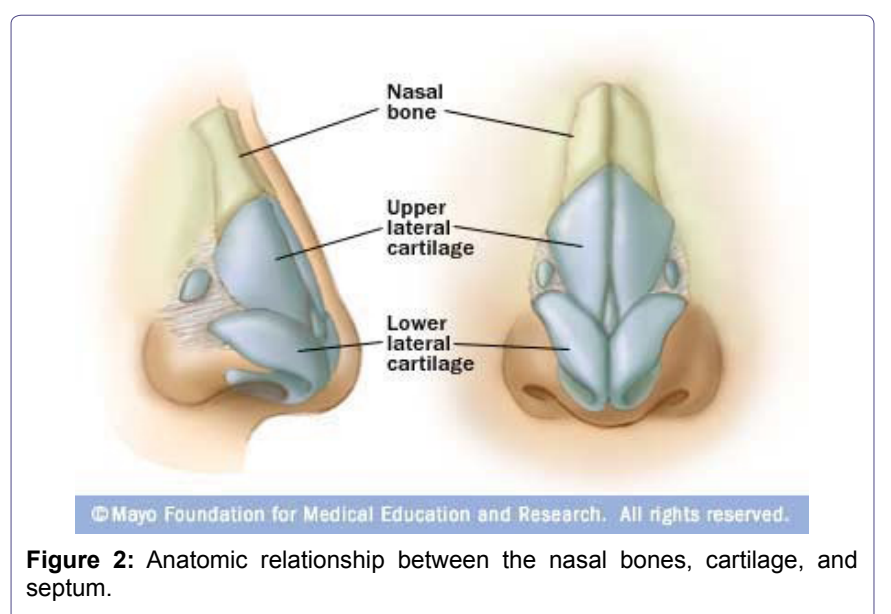

crura of the two lateral cartilages. The vomerian groove supports the inferior part and the ethmoid bone the superior and posterior parts.

Internal and external carotid arteries supply the nasal blood. The majority stemming is from the greater palatine, infraorbital and sphenopalatine branches of maxillary artery. Blood supply to the inferior and middle turbinate is provided by branches of the external carotid artery as well as the anterior and posterior ethmoidal branches of the internal carotid artery.

Epistaxis is the most common symptom of the nasal fractures, due to the mucosal injury derives commonly anteriorly from Kisselbach's plexus, located in the anteroinferior septal area. Posterior bleeding may result from both the sphenopalatine and/or anterior ethmoidal arteries. And the posterior bleeding control may be more difficult, which may require internal packing, balloon catheterization, interventional embolization.

The ophtalmic and maxillary branches of trigeminal nerve maintain the neural innervation of the nasal region. The infratrochlear nerve provides sensation to the skin of the upper nasal dorsum and sidewalls, and the anterior ethmoidal supplies the lower dorsum and tip [2]. The olfactory epithelium cells are derived from both the olfactory placode and the neural crest. This epithelium, which lines the upper regions of the septum, cribriform plate, superior turbinate, and sectors of the middle turbinate, is innervated by olfactory receptor cells, by fibers from trigeminal nerve and nervus terminalis (cranial nerve 0 ) and from the superior cervical ganglion. When an odorant enters the nasal cavity, it is absorbed into the mucus covering the olfactory epithelium. This mucus is derived largely from specialized Bowman's glands [12].

\section{History and Examination}

The clinical evaluation is the first base of the diagnose. A detailed patient history and examination is critical for the process. Proper patient selection and the true repair time is the first base of the duration. How it happened, time since injury, patient age may be determined carefully. There should be a priority to the patients requiring acute reduction.

The reason of the trauma should be learned (sport, weapon, fall, motor vehicle accident, etc.). The mechanism of trauma is very important to guess the direction of trauma and also velocity of the force. Physical alterations may result in typically low-energy lateral force transmission to the nose. The common traumas incur an in-fracture on the ipsilateral nasal bone, out-fracture on the contralateral side, and typically involve septal deformities. Motor vehicle and projectile-induced traumas are commonly resulting in frontal injuries [2].

Non-accidental nasal injuries especially in infants signify child abuse. For this reason the determination of the non-accidental nasal injuries is very important for legal procedures. Attention should be made to the patient for concomitant fractures that can be mortal [13].

For a proper treatment; the timing of injury is very important. Treatment procedures differ from patient to patient according to the injury time, within hours or weeks after the injury. In the initial hours after injury, before the diffuse edema onset, it is easy to determine the full nasal deformities. After the diffuse edema onset, the examination will be difficult and the reduction should be delayed until the edema disappearance (approximately 3 to 5 days) [14].

Previous nasal trauma or procedures should be learned. It is important to compare the posttraumatic nose with a previous photograph. And the patient should be asked for frequent use of over-the-counter allergy medications or inhalers [2].

Seizure disorders or other comprimising medical conditions may precipitate nasal trauma. The patients with epilepsy are more susceptible to facial injuries [15].

Nasal fractures are common among the elderly. Mechanism of the fracture, comorbidity, capacity for tissue repair and healing are the critical properties for these patients compared with younger patients. Increased falls and poor bone stock are major conspirators in nasal traumas in the elderly. These fractures tend to favor nonoperative management. The most important factor is the management of concomitant injury. For these concomitant injuries, there is a high rate of intensive care and mortality compared with young patients. The surgical procedures may be carried out when it is medically safe and quality of life of this population [16].

The detailed examination should consist of internal and external parts of the nasium. Both of the physical examination may give more information. Obvious nasal defects, malposition may be determined easily by an external examination. Furthermore soft tissue injuries such as lacerations, hemorrhage, and/or edema require internal control. The critical manuplation is nasal palpation. Presence of crepitus, tenderness, depression, step-off, nasal shortening, or widening of the nasal base are indicative for fracture diagnose [2].

Nasal fractures may be result of a minor trauma or a complicated trauma. If there is a complicated trauma, the physician should remember any other injury until it is ruled out [8]. Presence of a significant rhinorrhea should be evaluated for Cerebrospinal Fluid (CSF) leak by testing fluid levels of either glucose or ß-transferrin [17].

Attention should be made to the possibility of facial or mandibular fracture. Manually palpation is critical especially for all bony facial fractures, including the malar eminences, orbital rims, zygomatic arches, mandible, and teeth [8].

The management of nasal fractures in anticoagulant users is a difficult procedure. The bleeding risk is high in these patients, if the dose of anticoagulants is not lowered. On the other hand lowering the drug levels can increase the tromboembolism risk. The control of epistaxis is difficult in these patients when the surgery is decided. For example, in warfarin users the warfarin should be changed to low molecular weight heparin at least 4-5 days prior to surgery. INR should be less than 2 for proceeding with surgery. For the 
tromboembolism risk, heparin should be discontinued 6 hour before surgery and restarted within 6 hours after surgery [18].

Anosmi or hyposmia typically follows acute nasal trauma secondary to mucosal swelling, collection of blood and secretions, or internal derangements, but involvement of the cribriform plate should be considered and ruled out [10]. Patients should be evaluated for disturbance in sense of olfaction. Nasal trauma can cause disturbances in sense of smell. Olfactory dysfunction occurs most commonly as a result of upper respiratory tract infections, trauma, and chronic rhinosinusitis. Complete or partial loss of olfactory function may occur when the nasal passages are blocked, olfactory nerves are injured or there are contusions or hemorrhages in olfactory centers of the brain [19]. The disturbances in sense of smell can be mild, moderate, severe, or total loss. Quantitative testing of the sense of smell, which is easy to perform in the clinic is critical for determining the nature and degree of smell dysfunction experienced by the patients [12].

Decongestant spray and/or topical vasoconstrictive medication may be useful for internal nasal examination. A head lamp and a nasal speculum may help the surgeon to control details. For the proper examination, the patient should be seated position [2]. Endoscopic assisted examination may also give more information about the inferior turbinate, inferior meatus, septum and posteroinferior septal junction [20].

The most important problem is the possibility of a septal hematoma. The septal hematoma is a bloody compartment located between the cartilage and the perichodrium. Attention should be made to the correct treatment of the septal hematoma. Because inadequate drainage of the hematoma may result in infection and cartilage necrosis; then unavoidable final may be saddle nose deformity. A sterile drain may be left in place against reoccurence. Splints or sutures may be benefical to both sides of the septum to provide pressure and support, or anterior nasal packing also may be used.

\section{Imaging Studies}

Although considered minor, a nasal bone fracture has the potential to be a serious injury. Nasal fractures can be alone or in combination with other head and face injuries [21]. Diagnosis of nasal fractures mainly basis on clinical. The radiograph is the first step in evaluating nasal bone fracture. The sensitivity and specificity of the radiograph in nasal fracture have been discussed for a long years by researchers (Figure 3) [22].

Diagnosis of nasal fractures mainly basis on clinical. CT may be benefical in complicated nasal fractures together with other facial fractures, such as mandible fractures. Previous reports indicated that radiographic techniques were negative in $25 \%$ of patients with nasal bone fractures who needed surgical treatment and facial computed tomography of these patients has a great risk of radiation to the lens (Figure 4) [23].

High Resolution Ultrasonography (HRUS) is another radiologic choice. HRUS costs low, is simple technique and the most importantly has no ionizing radiation risk. HRUS has a great diagnostic value. It has been shown in various studies. CT gives detailed bony anatomical knowledge to the surgeon. It is not operator dependent. But; a nasal fracture line might be missed from the partial volume artifact effect. HRUS can show even a disruption of $0.1 \mathrm{~mm}$ in the nasal bone, and it is not possible to differentiate between acute and chronic fracture lines
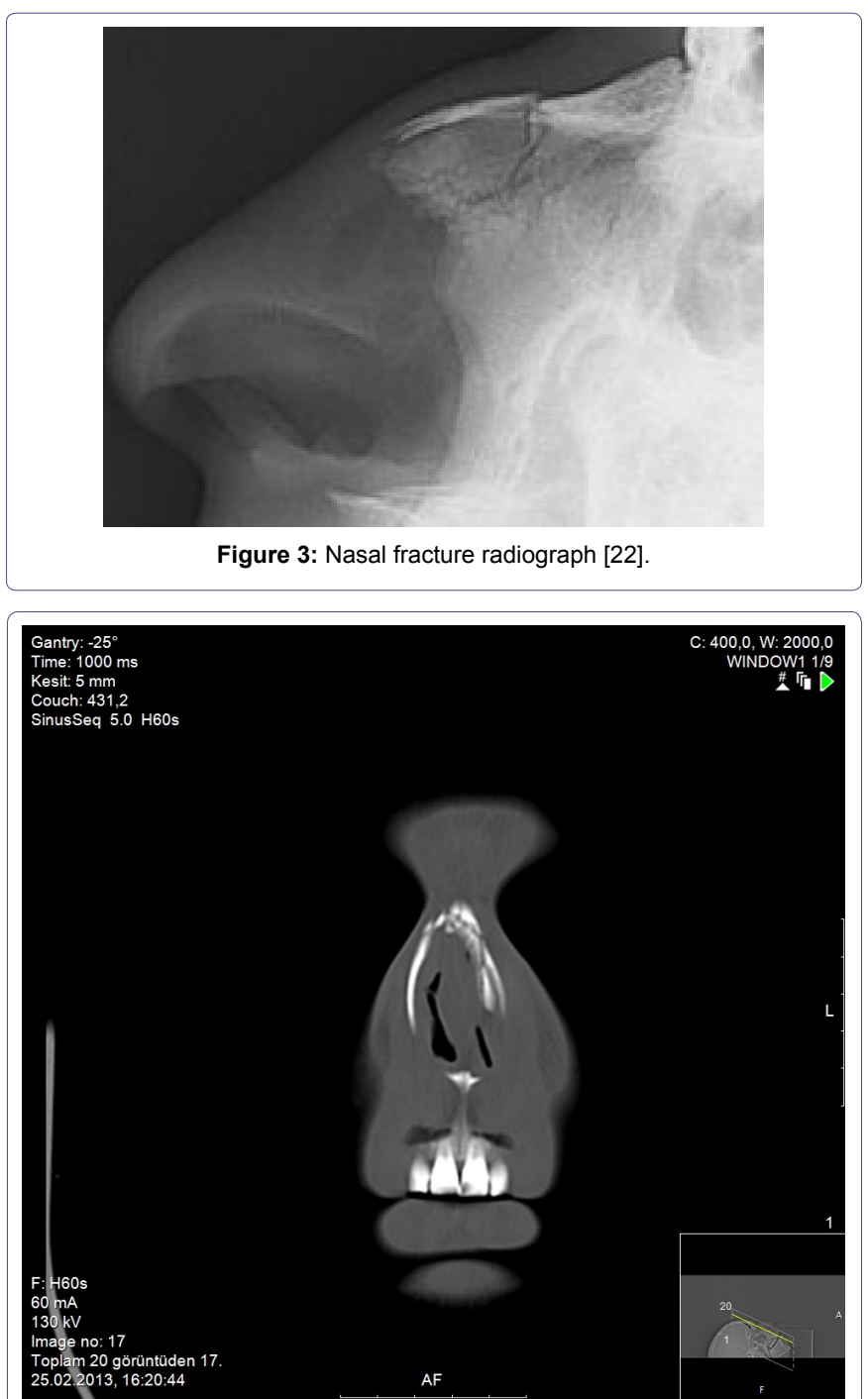

Figure 4: Nasal fracture CT scan.

by sonography. HRUS can tell the fracture time to the surgeon. HRUS is also a good indicator for cartilaginous structure and how it affected from the injury in comparison with conventional radiography [23]. The projected external shape of the nose is a disadvantage for HRUS. Existing linear probes have been too big to scan nasal bones, and an air gap could be seen between the lateral nasal bone and zygoma [24]. Nasal sonography has become popular in recent years because it provides detailed information about superficial areas in various imaging planes [22].

\section{Classification of Nasal Fractures}

Stranc and Robertson categorizated nasal fractures based on the vector of force and clinical findings. Murray et al., described nasal fractures based on pathologic criteria [2]. The senior author (S.S) reported a modified Murray classification heavily based on clinical findings (Figure 5).

Type I : Injury restricted to soft tissue

Type IIa: Simple, unilateral nondisplaced fracture

Type IIb: Simple, bilateral nondisplaced fracture

Type III: Simple, displaced fracture 
Type IV: Closed comminuted fracture

Type V: Open comminuted fracture or complicated fracture [2,17].

Nasal bone fractures was classified into six types according to CT scans

Type I: Simple without displacement.

Type II: Simple with displacement/without telescoping.

IIA Unilateral

IIAs Unilateral with septal fracture

IIB Bilateral

IIBs Bilateral with septal fracture

Type III: Comminuted with telescoping or depression [25].

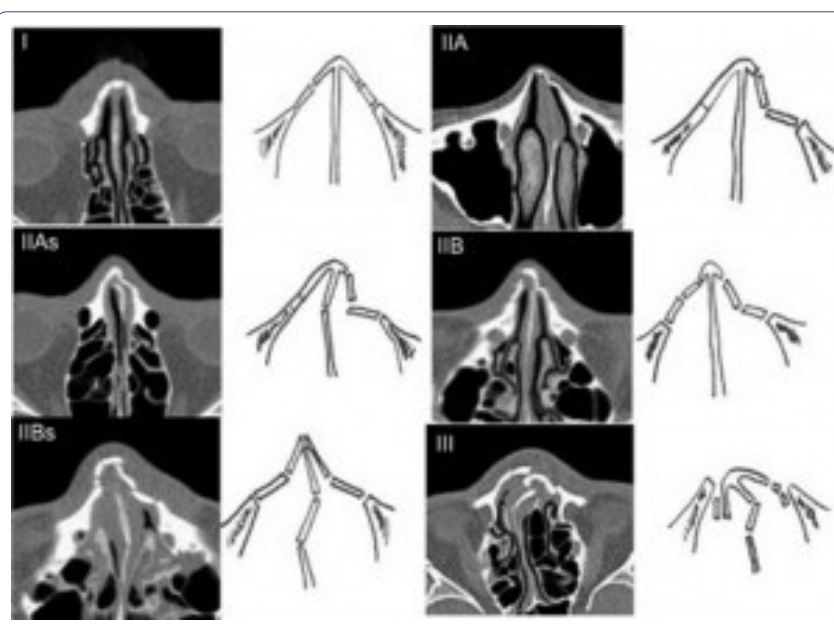

Figure 5: Classification of nasal bone fractures.

(I) Simple without displacement; (II) Simple with displacement/without telescoping; (IIA) Unilateral; (IIAs) Unilateral with septal fracture; (IIB) Bilateral; (IIBs) Bilateral with septal fracture; (III) Comminuted with telescoping or depression [25].

\section{Preoperative Management}

Treatment methods of nasal fractures differs from patient to patient according to some critical factors including, age of patient, trauma time, anesthesia type, necessity for acute versus delayed reduction, and surgical approach. Management of very young and very old patients is difficult. Conservative approaches should be preferred. The 13 to 14 year old children are proper for any septal surgery because of the facial growth centers. These growth centers complete their growth until these ages (Figure 6) [2].

\section{Anesthesia}

Pediatric patients may require general anetshesia, whereas most adults with severe nasal fractures can be reduced with a topical and infiltrative local anesthesia combination. Infiltrative anesthesia may be useful and used externally to the nasal dorsum and may be better tolerated over bilateral internal blocks [2].

Local anesthesia is consist of topical and infiltrative anesthesia. Lidocain (4\%) and either oxymetazoline or phenylephrine hydrochloride can be used. Three pledgets for 8 to 10 minutes for each nasal nostril is adequate. Specific focus areas should be along the dorsal septum near the anterior ethmoid nerve and artery, the middle turbinate proximal to the pterygopalatine ganglion, and the nasal floor adjacent to the nasopalatine nerve and sphenopalatine artery [17].

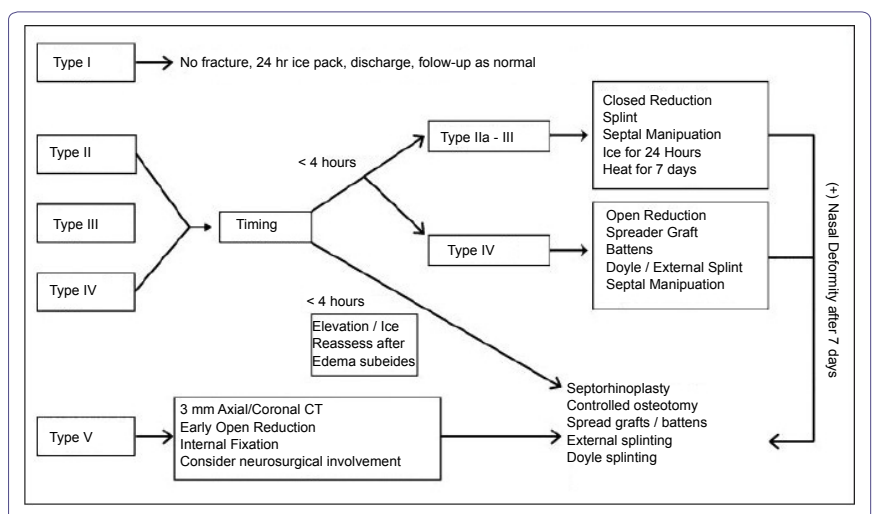

Figure 6: Algorithmic management approach of nasal trauma [2].

\section{Timing of Reduction}

Within 5 to 10 days after injury the nasal bones can become somewhat adherent and difficult to move. Fixation is usually observed within 2 to 3 weeks. The surgeon should choose a proper time for reduction when evaluation can be accurate and the bones are still mobile. The usual recommendation is that closed reduction be carried out within 3 to 7 days for children and within 5 to 10 days for adults [10].

\section{Closed Reduction}

It is suitable for the simple, non-comminuted nasal fractures. The critical manuplation is to maintain a force opposite to the vector of trauma. In this way a successful reduction should be performed. A Goldman elevator can be used to perform. It is useful for manual reposition of bones. Attention should be made to the cribriform plate for the injury possibility. The closed tecnique of the nasal fracture depends on the capability of reversing the vector of force that resulted in the injury. At first step is to reduce the nasal bone and second step reduction and stabilization of septum. A Goldman bar should be inserted into the nose up underneath the nasal bones and upward outward pressure exerted. The external digital manuplation and intranasal pressure maintain the adequate force to reduce the nasal pyramid.

Incomplete fractures may require osteotome mobilization for appropriate reduction [17].

\section{Open Reduction}

The method is necessary for patients can not be sufficiently managed with a closed reduction. Comminuted fractures with severe loss of nasal support, severe septal injuries, need to be exposured by open tecniques. Open tecniques may provide a detailed, excellent exposure. The process allows easier anatomical examination and reformation of nasal structures. Adequate exposure of septum, dorsum and tip provides a successful operation [2]. In severe nasal injuries early full septorhinoplasty method is necessary, because the result of closed reduction are unsatisfactory and an early correction should be performed, before scarring has taken place [26]. Incidence of deformities occured after closed reduction of a nasal fracture that require rhinoplasty has been reported to range from $14 \%$ to $50 \%$ [27-29]. As a result of nasal fracture, the deviation of the nasal septum places stress on the nasal bones, this causes the nasal bones to displace after reduction. In summary the nasal bones follow the position of septum [30]. 


\section{Splinting and Packing}

Both open and closed tecniques require splinting postoperatively. Splints should be internal and external. Doyle nasal splints are useful for internal splinting. They maintain internal septal stabilization and prevent synechiae after surgery [31]. Extranasal splinting maintain stabilization of reduced bones and cartilage, often after an open surgery. Packing is an old method, is used less frequently now. It is being accused for postoperative pain, discomfort, soft palate laceration, septal perforation, and even toxic shock [32,33]. Intranasal splinting should be lasted for 2 to 3 weeks postoperatively and packing should be no longer than 72 hours.

Follow-up should be 6 to 12 weeks postoperatively. This time is critical for structural integrity of the nose. The patient should be evaluated for the possibility of nasal collapse, loss of tip support. A future septorhinoplasty may be necessary for these situations. Cartilaginous spreader graft may be useful fot these cases. The rib graft can bu used as a columellar strut graft to improve and maintain projection in the loss of tip support. Bilateral batten grafts are becoming popular for maintaining nasal straightness [2].

\section{Conclusion}

The most important aim of treatment of patients with nasal bone fractures is to return to the before condition after the injury. The clinical evaluation and management should be individualized for the type and severity of injury. It is timing of shrewd. A proper assessment should maintain a proper healing, breathing and smelling. By this way the difficult, exhausting adventure of the patient may result in success.

\section{References}

1. Renner GJ (1991) Management of nasal fractures. Otolaryngol Clin North Am 24: 195-213.

2. Kelley BP, Downey CR, Stal S (2010) Evaluation and reduction of nasal trauma. Semin Plast Surg 24: 339-347.

3. Dingman RO, Natvig $P(1969)$ The Nose. In: Natvig $P$ (eds.). Surgery of Facial Fractures. Philadelphia, PA: Saunders.

4. Murray JA, Maran AG (1980) The treatment of nasal injuries by manipulation J Laryngol Otol 94: 1405-1410.

5. Bailey BJ, Tan LKS (1998) Nasal and Frontal Sinus Fractures. In: Bailey BJ (eds.). Head and Neck Surgery-Otolaryngology. ( $2^{\text {nd }}$ edn). Lippincott-Raven, Philadelphia, PA, USA.

6. Azevedo AB, Trent RB, Ellis A (1998) Population-based analysis of 10,766 hospitalizations for mandibular fractures in California, 1991 to 1993. J Trauma 45: 1084-1087

7. Çil Y, Kahraman E (2013) An analysis of 45 patients with pure nasal fractures Ulus Travma Acil Cerrahi Derg 19: 152-156.

8. Kucik CJ, Clenney T, Phelan J (2004) Management of acute nasal fractures. Am Fam Physician 70: 1315-1320.

9. Vora NM, Fedok FG (2000) Management of the central nasal support complex in naso-orbital ethmoid fractures. Facial Plast Surg 16: 181-191.

10. Arden RL, Mathog RH (1993) Nasal Fractures. In: Cummings CW, Otolaryngology Head and Neck Surgery. Mobsy-Year Book Inc., St. Louis, MO, USA.

11. Rubinstein B, Strong EB (2000) Management of nasal fractures. Arch Fam Med 9: 738-742.
12. Doty RL, Kamath V (2014) The influences of age on olfaction: a review. Front Psychol 5: 20.

13. Roberton DM, Barbor P, Hull D (1982) Unusual injury? Recent injury in normal children and children with suspected non-accidental injury. Br Med J (Clin Res Ed) 285: 1399-1401.

14. Rohrich RJ, Adams WP Jr (2000) Nasal fracture management: minimizing secondary nasal deformities. Plast Reconstr Surg 106: 266-273.

15. Nonato ER, Borges MA (2011) Oral and maxillofacial trauma in patients with epilepsy: prospective study based on an outpatient population. Arq Neuropsiquiatr 69: 491-495.

16. Zelken JA, Khalifian S, Mundinger GS, Ha JS, Manson PN, et al. (2014) Defining predictable patterns of craniomaxillofacial injury in the elderly: analysis of 1,047 patients. J Oral Maxillofac Surg 72: 352-361.

17. Higuera S, Lee El, Cole P, Hollier LH Jr, Stal S (2007) Nasal trauma and the deviated nose. Plast Reconstr Surg 120: 64-75.

18. Wazir S, Khan M, Ud Din Q (2012) Management of a Maxillofacial Trauma Patient on Warfarin. Pakistan Oral \& Dental Journal 32: 36-38.

19. Akdogan Ö, Selçuk A, Gürbüz D, Dere H (2008) Analysis of Simple Nasal Bone Fracture and the Effect of it on Olfactory Dysfunction. Kbb-Forum 7: 68-70.

20. Park CH, Kwon TK, Lee JH, Hong SJ, Joung HH, et al. (2009) Endoscopically assisted reduction of nasal bone fractures. Rhinology 47: 301-304.

21. Bartkiw TP, Pynn BR, Brown DH (1995) Diagnosis and management of nasal fractures. Int J Trauma Nurs 1: 11-18.

22. Chou C, Chen CW, Wu YC, Chen KK, Lee SS (2014) Refinement treatment of nasal bone fracture: A 6-year study of 329 patients. Asian J Surg .

23. Mohammadi A, Ghasemi-Rad M (2011) Nasal bone fracture--ultrasonography or computed tomography? Med Ultrason 13: 292-295.

24. Lee MH, Cha JG, Hong HS, Lee JS, Park SJ, et al. (2009) Comparison of high-resolution ultrasonography and computed tomography in the diagnosis of nasal fractures. J Ultrasound Med 28: 717-723.

25. Hwang K, You SH, Kim SG, Lee SI (2006) Analysis of nasal bone fractures; a six-year study of 503 patients. J Craniofac Surg 17: 261-264.

26. Fernandes SV (2004) Nasal fractures: the taming of the shrewd. Laryngoscope 114: 587-592.

27. Ziccardi VB, Braidy H (2009) Management of nasal fractures. Oral Maxillofac Surg Clin North Am 21: 203-208, vi.

28. Waldron J, Mitchell DB, Ford G (1989) Reduction of fractured nasal bones; local versus general anaesthesia. Clin Otolaryngol Allied Sci 14: 357-359.

29. Crowther JA, O'Donoghue GM (1987) The broken nose: does familiarity breed neglect? Ann R Coll Surg Engl 69: 259-260.

30. Green KM (2001) Reduction of nasal fractures under local anaesthetic. Rhinology 39: 43-46.

31. Doyle DE, House LF, Hall WP (1977) Description of a New Device: An Intranasal Airway/Splint. Laryngoscope 87: 608-612.

32. Fairbanks DN (1986) Complications of nasal packing. Otolaryngol Head Neck Surg 94: 412-415.

33. Stucker FJ, Ansel DG (1978) A case against nasal packing. Laryngoscope 88: 1314-1317. 